\title{
An Object Oriented Database Approach for Supporting Hypertext
}

\author{
Bing Wang \\ Engineering Department \\ University of Cambridge \\ CB2 1PZ, United Kingdom \\ bw@uk.ac.cam.eng
}

\author{
Peter Hitchcock \\ Computer Science Department \\ University of York \\ Y0I SDD, United Kingdom \\ ph@uk.ac.minster.york
}

\author{
Tony Holden \\ Engineering Department \\ University of Cambridge \\ CB2 IPZ, United Kingdom \\ holden@uk.ac.cam.eng
}

\begin{abstract}
Almost all hypertext systems do not use DBMS as their information base. This paper describes a prototype, called InterSect, which is a hypertext system integrated with an object oriented database. The data model of InserSect is an object oriented hypertext dath model. This model will be described by using $Z$ specification language. There are three main fealures of the InterSect approach. First, IneerSect is designed in 2 lully object oriented way. Second, some important mechanisms such as versioning and composition of the next generation bypertext systems are supporied by InterSect. Third, InterSect supports a conventional database view logether with a bypertext view of the database contents. We will use as an example the software documentation environment in order to illustrate these characteristics of InterSect.
\end{abstract}

\section{Introduction}

Hypertext has been widely accepted as a useful tool in documentation reading and writing environments. It has simple and powerful abilities to browse objects which allow hypertext to be used in a variety of fields including medical, education and military. The fundamental mechanisms and concepts of hypertext are not complicated. Nodes are used as the information containers to store different types of information such as text, image and sound. Links are the semantic relationships among nodes. In the hypertext application environments, nodes are nomally represented as the active areas on a computer screen such as windows or rectangles in a window. Links are the menu style operations which can give users the chance to jump from one node to another, navigate the hyperspace and find the target information they want.

In recent years, the appiication areas of hypertext have been explored. Some research have showed that hypertext can be extensively used in some complicated documentation environments, for instance, office automation and software documentation environment[1,2]. In these application areas, situations are far more complicated than simply browsing and storing documents. This is because not only do large quantities of complex objects need to be stored but also many complex relationships among objects should be maintained[3]. However, it is difficult to support these complicated documentation environments by only relying on the current mecharisms of hypertext systems. Halasz[4] listed seven reasons why the current hypertext systems lack the ability to support complex applications although his view only focused on the NoteCard weak points. 
In Cambridge and York, we have developed a prototype system, InterSect, which uses the object-oriented database DAMOKLES as its information base. Such a combination can be used to support complex documentation environments. In this paper, we will discuss the rationale of our approach in the following three aspects: firstly, a formal data model of combining OODB and hypertext systems is presented in particular emphasizing the definitions of composition and versioning of node types and link types. Secondly, one of the advantages of databases is that they can easily manage complex relationships between entities. On the other hand, links of hypertext represent the semantic relationships between nodes. It is therefore important for us to give a precise clarification between relationship definition and link definition. We will discuss the differences between these two definitions in the InterSect approach. Thirdly, we have found that it is important to decide how "big" a node is, i.e. the "chunk size" of information contents, when using hypertext to manage large numbers of different types of documents. We will discuss the definition of node size in the InterSect approach when it is used in complex documentation environments. The paper is organized as follows: Section 2 represents a formal extended hypertext data model of InterSect. Section 3 discusses some design issues of InterSect. Section 4 briefly reviews some related works.

\section{An Extended Hypertext Data Model}

Nodes and links are the basic constructs of the current hypertext systems. However, many hypertext systems adopt a hierarchical composition mechanism to construct a more complex node type, such as the stack used in HyperCard as the named collection of cards[5], or the encyclopedia used by Hyperties to represent a related set of articles[6]. As mentioned by Halasz[4], current hypertext systems cannot deal with complex situations because they lack a composition mechanism to represent a group of nodes as an unique object and they do not have a versioning mechanism to maintain history. Composition and versioning are basic and important aspects in complex documentation applications. The extended hypertext data model called InterSect_DM presented in this section deals with these two problems in particular. In the following discussion of the data model we will use the $Z$ notation $[7,8]$ to illustrate the fundamental mechanism of the data model.

First, we give some basic type definitions.

\section{- Object identity}

Object identity is the property of an object which can be used as an unique identifier to distinguish objects from one another. Each node defined in InterSect_DM has a unique identity created by the underlying information base. All object identifiers belong to the given set of Object IDentifiers [OID].

\section{- Attribute}

In InterSect approach, each node type can attach a number of attributes to illustrate the property of the defined node types. We use the given set [Attribute] to represent all the possible attributes of the defined node types.

\section{- Contents of node}

The contents of a node can be a combination of different types of information, text, image, sound, exc. In order to illustrate all the different contents of node types, we use the given set [Multimedia] to represent all possible information contents of node types. 


\subsection{Structured versioning node types}

The node types defined in InterSect_DM have the following three important features:

- All node types belong to two types: control node type and normal node type.

- All node types are defined as structured node types with versions;

- Each node type is recursively defined. That is, a node can contain a number of subnodes.

In the current hypertext approach, nodes only represent the concept of information holder. This means that a node must be an information container, otherwise it is not a node. Such a constraint restricts us in our representation of the structures of complex documentation environments. This weak point is overcome by the InterSect approach. We allow users to define a node without contents, called a structured control node, which belongs to the structured control node type. The structured control node type plays two significant roles in a complex documentation application environment:

\section{(1) Existence constraints[9]}

It is used as the existence constraint to determine the existence of other nodes in order to maintain referential integrity. ${ }^{(1)}$

\section{(2) Semantic linkage}

It acts a semantic connection to link two node types.(1)

A normal node defined in InterSect is similar to the conventional definition of the node defined in the current hypertext systems. However, it differs from the current hypertext node definition because it allows versioning and is structured. One of the main advantages of the node type defined in InterSect_DM is that it allows users to define any complex structured node type which may or may not have versions according to the needs of applications.

Whether the defined nodes have corresponding contents or not nodes defined in InterSect_DM belong to the $Z$ schema definitions either With_Contents or Without_Contents. Strictly speaking, structured control versioning nodes belong to the $Z$ schema definition of Without_Contents and structured versioning nodes belong to the $Z$ schema definition of With_Contents.

Nodes defined in InterSect_DM are structured nodes. This implies that any node can contain zero or a number of structured nodes which may or may not themselves have versions. The node type definition of current hypertext systems is the special case of a node type in InterSect_DM which only contains zero structured nodes. In the following $Z$ schema definitions, we use free type definition of Z[8] to illustrate the characteristics of structured control node type and structured node type, which both are the versioning node types. (2)

\footnotetext{
(1) This can be found in the example used in the appendix.

(2) If the version number of a structured node is zero, this node is a generic node. i.e. it has no corresponding versions. null_struct represents the null structured node type, i.e. no structured nodes.
} 
- Structured versioning node type without contents

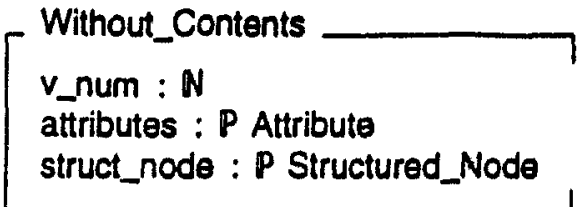

Without_Contents: represents structured versioning nodes which do not have contents attached. If the v_num equals zero, it represents a generic node. All such nodes can contain zero or a number of Structured_Node.

- Structured versioning node type with contents

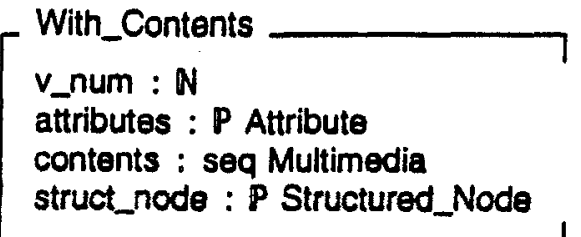

With_contents: is the same as the schema definition of Without_Contents, except that structured versioning nodes now have corresponding contents.

- Structured versioning node type

Structured_Node ::= null_struct I structured_control_node \&Without_Contents» I structured_node «With_Contents»

Structured_Node: says that Structured_Node is either a structured control versioning node denoted by the constructor, structured_control_node or a structured versioning node represented by the consmucior, structured_node or null structured node, null_struct.

\subsection{Link type and relationship type}

Links play a semantic conrection role in hypertext systems. In hypertext systems, links are the machine supported direct connections between nodes[10]. Because they are machine supported, they are usually represented as the operations which allow users to navigate when browsing in hyperspace.

Relationship is a concept heavily used in system design and analysis environments when modelling the real world, e.g. the entity-relationship model[11]. There are many different types of relationships in a complex application environment and it is difficult for human beings to use limited relationship types to represent every situation in complex 
application environments. These complex relationships can be classified into two classes: intra-relationship and inter-relationships.

Intra-relationships describe the internal structure of a particular object, whereas inter-relationships reveal the connection between different objects. Such a classification of relationship types means that most links defined in current hypertext systems play the role of the intra-relationships which reveal the semantic meanings of the document structure. (3) It is therefore important for the integration system to have the capabilities to present intrarelationships as well as inter-relationships. This the InterSect prototype does.

The data model of InterSect, InterSect_DM, allows the user to define any explicit named relationship between two structured nodes. The defined relationship is controlled by the information base, DAMOKLES, an object oriented database management system. In addition, a relationship defined in InterSect_DM has its own following special purposes:

\section{(1) High level integrity constraints}

One of the basic objectives of a DBMS is that it can guarantee that stored data satisfies semantic assertions, i.e. integrity constraints, which can be either at the individual data level or at data type level. Since the structured node types describe the properties of individual nodes, the relationship type defined between two structured node types guarantee that all individual nodes also have the same semantic constraint described by the corresponding relationship type. Therefore, we need not to establish every semantic constraint between individual nodes as does the current hypertext approach. This is especially useful when constructing a complex documentation environment.

\section{(2) Semantic constraints among structured nodes}

The structured control node types defined in InterSect_DM do not have corresponding contents attached. This means that we cannot make any semantic links within a structured control node. However, as we described before, structured control nodes play an important role in the InterSect approach. It is necessary to build up some semantic relationships among the structured nodes in an application environment. This can be done by using relationship type definitions.

The $\mathbf{Z}$ schema definition of the relationship type defined in InterSect_DM represents a named relationship between two structured node types.

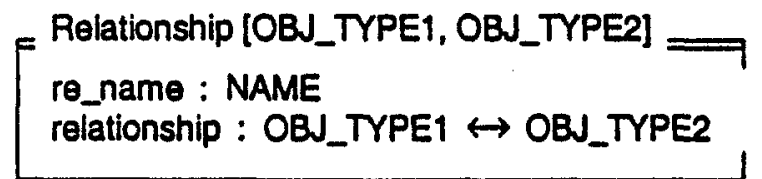

Relationship:
nays that OBJ_TYPE1 and OBJ_TYPE2 have a
ment of the given set NAME which represents
all the possible names of the defined rela-
tionship types. OB__TYPE1 and OBJ_TYPE2 are

\footnotetext{
(3) Although some links deal with the organization of nodes such as is-a links and has-a links[12], we think these links still have the limited capabilities to represent the inter-relationships between entities. Is-a links, for example, only illustrate the membership relation between two objects whereas has-a links only describe the characteristics of the defined nodes. Because of the limiled capabilities of the links of hypertext systems, it is difficult to use current bypertext systems in complex documentation environments when thousands of different types of documents are needed to be maintained[13].
} 


\section{of the Structured_Node type.}

The link types defined in InterSect_DM are close to the definition of links of the most current hypertext systems. In order to more easily extend the link structure of InterSect_DM in the future, we defined the InterSect_DM links in an object oriented way, that is, the defined links are all the subtypes of the supertype Link.(4) At the moment we only defined two different link types, annotation links and referential links. Our aim is to use these two link types as examples of the use of link structures in an integrated hypertext system. More link types, such as a zooming link to increase detail or a view link which will restrict the information presented, will be implemented in later development of InterSect prototype. ${ }^{(5)}$

An annotation link allows users to make any annotation within the contents of a node. A referential link is a link that users use to make a referential connection from within the contents of one node to other nodes, which may or may not be the same type. More importantly, the links defined in InterSect_DM are versioning links. This means that the same links can be made several times from the same piece of data within the contents of a node. This is illustrated by the following diagram:

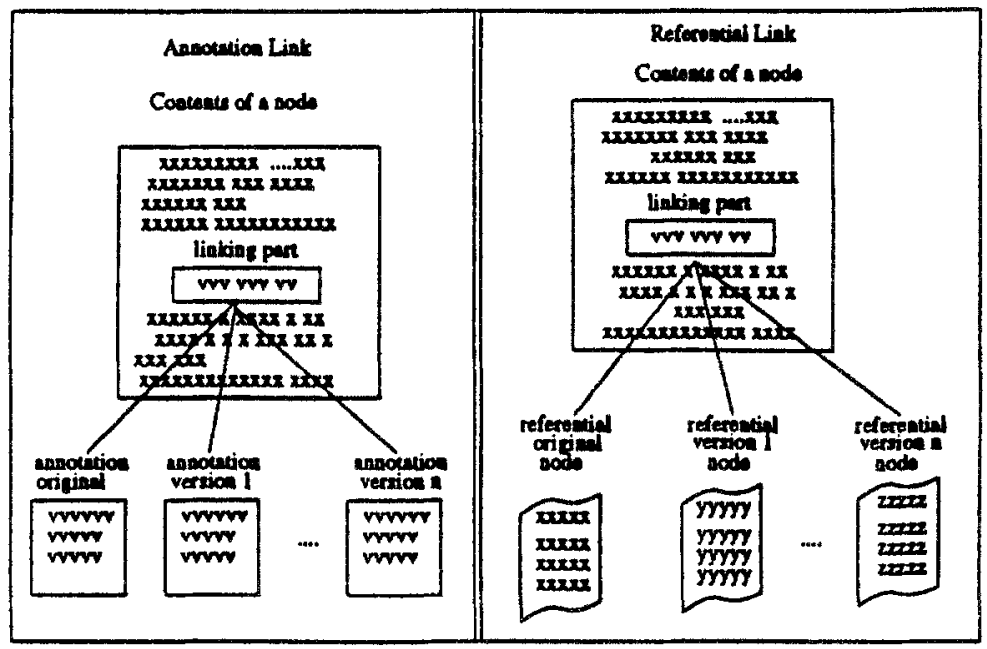

Fig. 1. Annotation link and referential link in InterSect_DM

The following are the corresponding $Z$ sechma definitions. We firstly define the Linking_Part $Z$ schema definition which describes the part within the contents of a node. i.e. the source of the hypertext links. .

\footnotetext{
(4) In the implementation, the link structure is structurally defined and captured by a link schema called Hyper defined in DAMOKLES DDL.

(5) Because we bave used the object oriented design method to design the InterSect, it will not be difficult if we add more link types to InterSect. This can be described as follows: first, the new defined link type will be defined in DAMOKLES DDL as a subtype of supentype Link; second, the corresponding programming source code describing the operations of the new defined link type will be designed as a module; third, this module is then linked to the public interface of the Node + Link Manager.
} 


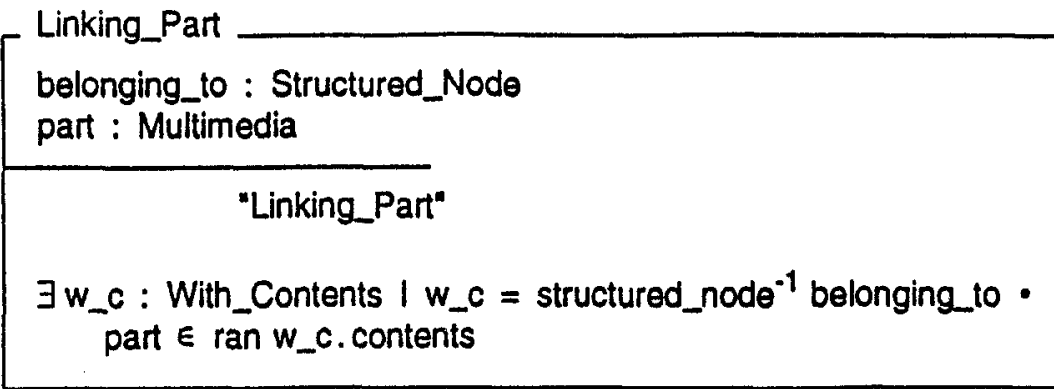

Linking_Part ensures the node which can make links must be a node with contents and the linking part must be within such cont its.

- Annotation link type

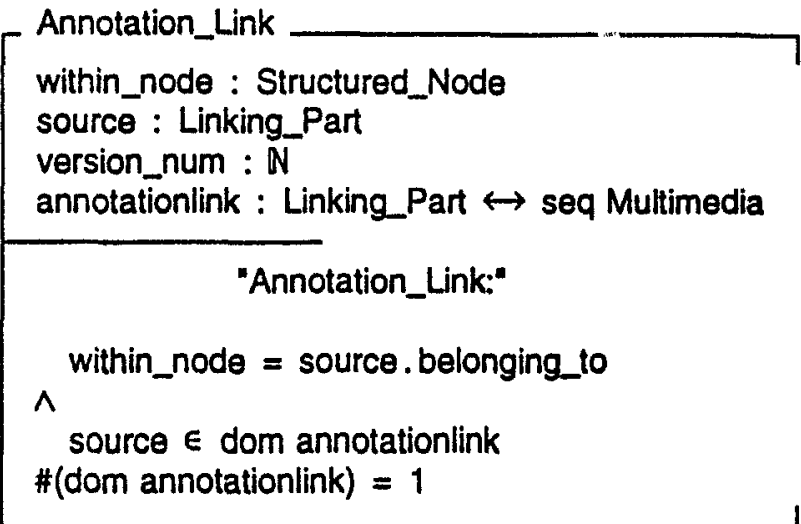

Annotation_Link: represents that a single linked part, i.e. the source of a link, is within the contents of the selected node. The targets of an annotation link are objects belonging to the type Multimedia, the fact that this is a sequence means that the annotation can be a composite object. version_num describes whether the annotation link is an original link or a versioning link. If the version_num equals zero, it presents the original annotation link, otherwise it represents a versioning link. The different version instances of a link will all have the same source part. 
- Referential link type

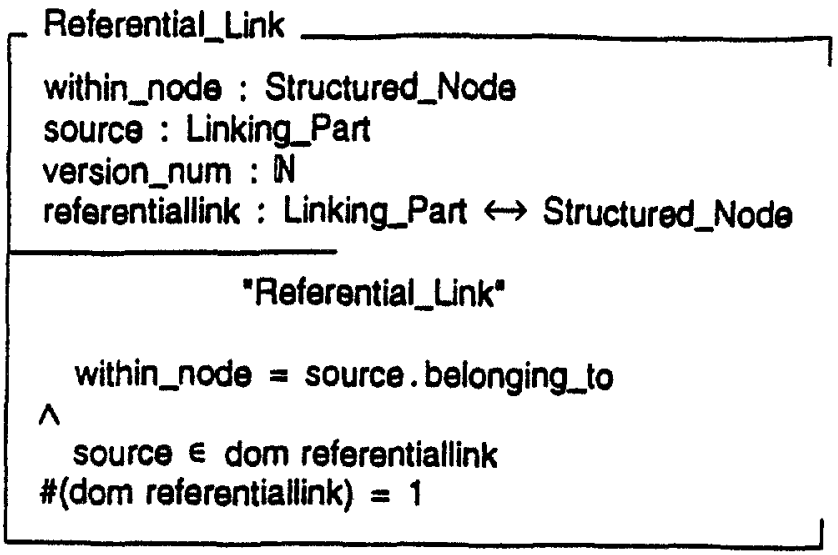

Referential_Link: is the same as annotation link definition
except that the linked part within the
contents of a node may be linked to
several nodes which may belong to the
same or different node types.

- Link type

Link ::=

annotation_link « Annotation_Link \$

referential_link «Referential_Link»

Link: represents that Link is a structured type which is composed of either an annotation link denoted by the constructor, annotation_link or a referential link denoted by the constructor, referential_link.

\subsection{Node size in InterSect_DM}

When using bypertext systems to manage different types of documents, we should first decide on the size of the node, i.e. how much information should be stored in a node. The key question here is whether the information to be stored is naturally divided into the size of node defined by the hypertext system. As Brown[14] described, "...if the underlying information is naturally divided up inso separate card-sized units, then the card-based approach is the best. If not, dividing the material into cards adds an extra and unnecessary level of complexity."

We have found that it is ineficient to restrict or pre-decide the node size before the application starts. So, in the InterSect approach we allow users to decide how "big" the node is. That is, the user decides the type and number of nodes, the size of nodes and the relationships among the nodes. All this information is defined by the $Z$ schema definition, Global_Application_Environment. ${ }^{(6)}$ Because the node type defined in InterSect_DM is

\footnotetext{
(6) In the application environment, the documentation structure is described in DAMOKLES Data Definition Language. This is illustrated in the appendix.
} 
strucured, it is easy for InterSect_DM to model any bind of text information whether they are suitable for dividing up into card style units or not.

Research has already shown that structured documents are relatively easily for making hypertext applications $[14,15]$. Those documents such 2s ODA[16] and IEE Software Documentation Guidelines[17] are structured and well defined. However, in the actual applications, we find that even if the documentation is well defined and structured, hundreds of structured objects still need to be defined and maintained. Further more, how to construct a structured node is another problem in constructing a hypertext document. In IEE Software Documentation Guideline[17], for example, the User Requirements Specification Document has four parts: Overview, System Objectives, System Interfaces and System Environment. Each part is composed of several subparts. These are also structurally organized, for instance. System Objectives consists of these fragments: System Modes, System Functions, Operational Requirements, Equipment, Operator Control Consoles and so on. Therefore, there are several design decisions to make. There could be one structured node which contains all the information of User Requirements Specification, or it can be a complex structured node consisting of several subnodes which in turn are struc. tured nodes.

The flexible way of determining the node size of Intersect_DM gives users the chance to decide the node size for thernselves. This means that, a node can be a small node which only contains a sentence or it can be a big node which contains thousands of paragraphs. At the same time, hypertext links can be made on any part within the contents of a node as required. Based on the above definitions, a user global application can be modelled and described by the following $\mathbf{Z}$ schems which illustrates that the defining application environment consists of nodes, links and relations.

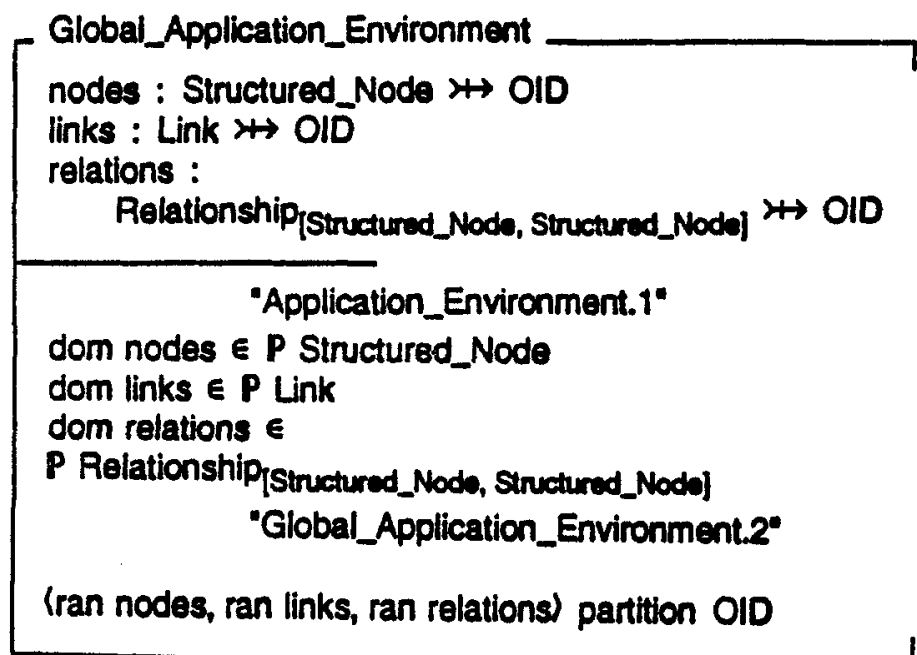

$\begin{array}{ll}\text { Application_Environment.1 } & \begin{array}{l}\text { ensures that the nodes, links and } \\ \text { relations defined by users must } \\ \text { exist in DAMOKLES information } \\ \text { base. } \\ \text { ensures that the set of object } \\ \text { identifier is disjoint and }\end{array}\end{array}$




\section{partitioned by the created \\ objects identifiers.}

\section{The Design Issues of the Prototype}

In order to test the above extended hypertext data model, we have developed a prototype system called InterSect. InterSect is implemented as C++ program on top of DAMOKLES on a Sun 3/50. In the appendix, we use a module example to demonstrate the main features of InterSect. For the limited length of the paper, we cannot present all the detailed operation definitions here. They run to some twenty pages of $Z$ schema definitions. In the following paragraphs, we focus on the following issues to discuss the design strategy of the InterSect prototype:

- It is an application independent prototype;

- It is designed in an object oriented way;

- It has an object oriented architecture;

The main aim of this research is to find out the possible way of combining an object oriented database with a hypertext system to support any complex documentation environment. Therefore, the application areas of InterSect should not be restricted to any particular application domain. Keeping this aim in mind, we decided to implement InterSect to be driven by the schema definition of the application. This definition uses the data definition language of DAMOKLES.

The second feature of Intersect is its object oriented design methodology. The implementation language is an object oriented programming language $\mathrm{C}++$. The editing tool Frame and information base DAMOKLES are all designed in an object oriented way[18]. As shown in the appendix, the editor, Frame, is defined as a C++ class, Frame, which captures all the editing information of the inputing text. Whenever the editor is called, an instance of this class is created. After leaving editor mode, the link and node information are sent to the information base DAMOKLES through the public interfaces defined in the Frame class. This is the same for other class definitions such as the Menu class for dealing with querying information and the ShowPage class for showing individual nodes and links. There are nearly ten such classes defined in InterSect. Three software design criteria have guided the design of the source code of InterSect. These are:

- Modularity

In order to let the source code of InterSect to be easily extended and developed, the InterSect interface operations and the operation of the information base are separated. Any changes in the interface will not influenc the design of the operations defined in the information base. On the other hand, all the components of the prototype which are shown in figures 3 and 4 are organized as autonomous and coherent modules. This means that if any unexpected error happens the error message will be displayed either on the terminal or on the interface screen. For example, if something is wrong in the application schema analysis stage, InterSect will show the following error message:

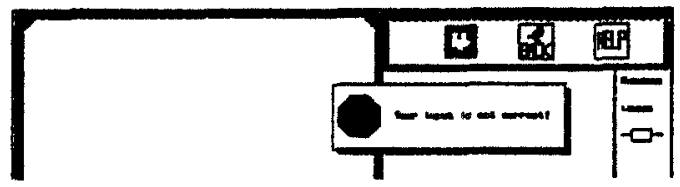

Although the aim of this error message is to tell users that unexpected things have happened in schema analysis stage, this message also shows that something is wrong with the 
schema analysis module if the user's inputing is correct. There are nearly eighty such error messages defined for the InterSect prototype. This made development easier.

\section{- Extendibility}

When we designed InserSect, we tried to fully use the object oriented mechanism in C+t. This is the class definition. Class is the main contribution of the $\mathrm{Ct+}$ programming language. We defined InterSect structures to be class strcutures as much as possible. This means that we can easily add more functions by using the inheritance mechanism in $\mathrm{C}++$, i.e. a subclass based on the defined classes in future development.

\section{- Reusability}

The object oriented database used to support InterSect, DAMOKLES is a research prototype developed by the University of Karlsruhe. West Germany[19,20]. There are many significant characteristics in the DAMOKLES database such as complex structured object type definition, versions and high level application definition. However, it is not very easy to use. Only DAMOKLES was available to us when we developed InterSect, so the design strategy we use is to let the interface part of InterSect be independent from the underlying information base and to provide standard public interfaces between the interface part and information base in order to more easily change the information base in later development.

As well as the above, the architecture of InterSect is an object oriented architecture. Different abstractions of the InterSect architecture can be found throughout the whole prototype. The following diagram is the first abstract view of the InterSect architecture in the Unix environment.

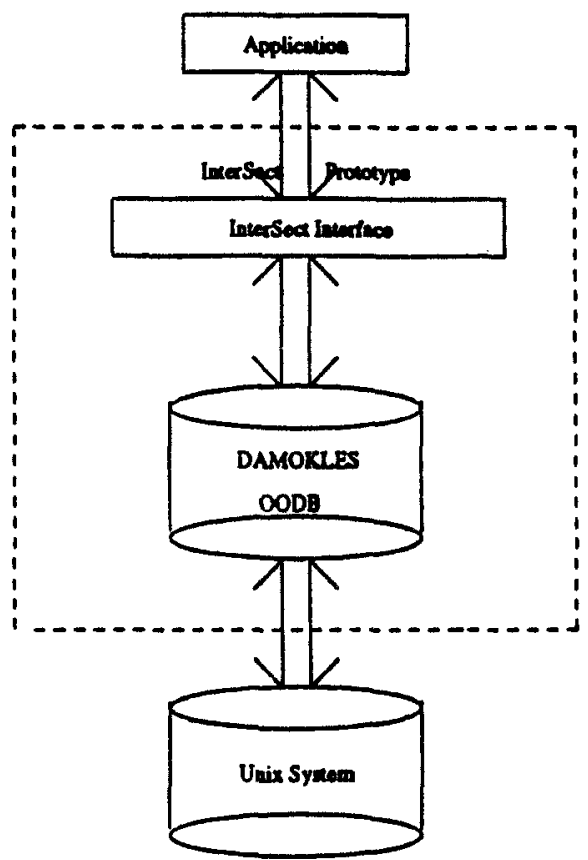

Fig. 2. Abstraction of InterSect architecture

For InterSect itself, it can be abstracted into five main parts as shown in figure 3. 


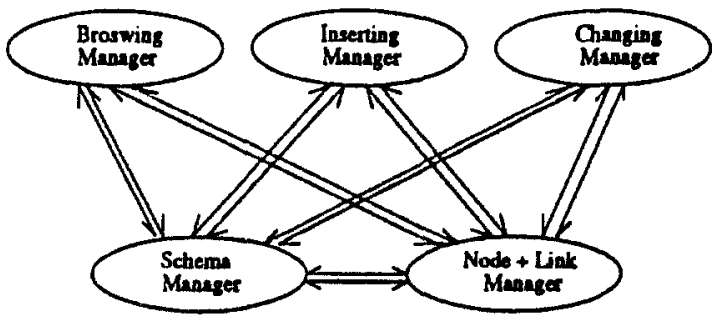

Fig. 3. Functional components of InterSect

The function of the Browsing Manager is to map hypertext data into a visual representation by using menu operations; the Node + Link Manager supports the manipulation of hypertext data between the application and DAMOKLES; the Inserting Manager deals with inputting data; the Changing Manager is responsible for changing existing data, and the Schema Manager deals with inputing schema definitions and passes the result of analysis to the Browsing Manager, Inserting Manager, Changing Manager and the Node + Link Manager. In addition, the third abstraction is the abstract view of these managers' architecture.

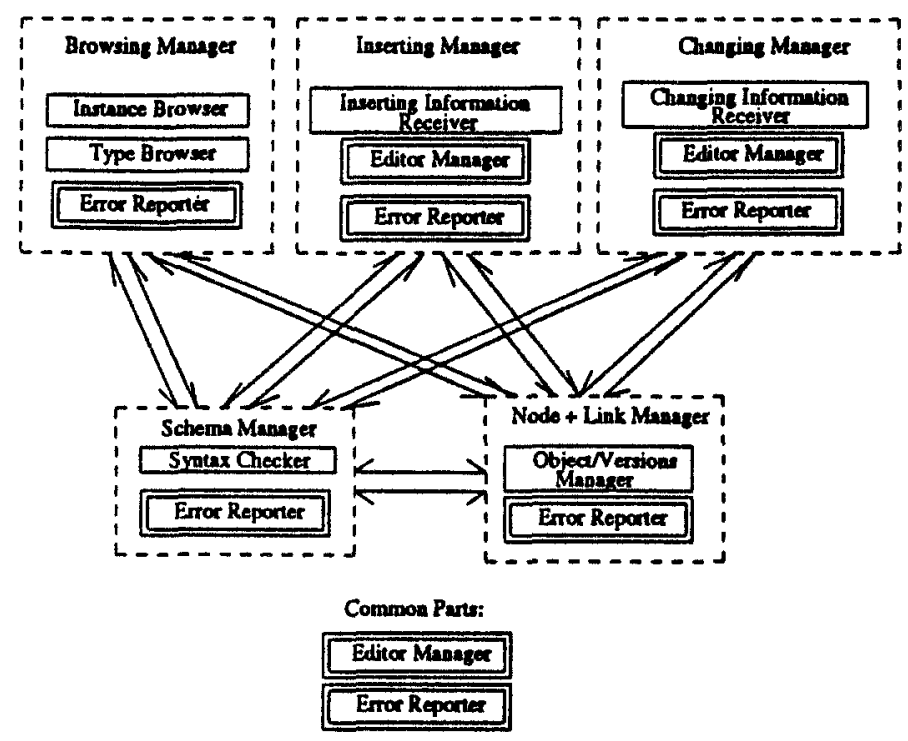

Fig. 4. Abstract view of each manager of InterSect

The common parts in figure 4 indicates that the implementation mechanism in these parts is the same. For example, the Error Reporter reports different error messages according to different situations. In inserting and changing models, the implementation mechanism of the Editor Manager is the same except that the editor will be used to inserting a contents of a node in inserting model whereas in changing model the editor is used to changed a conetents of a node. Each subcomponent of these managers is independent and selfcontained. They are connected only by sending and receiving messages from each other. 


\section{Related Works}

In current hypertext research, almost all the commercial hypertext systerns do not use database systems. They are built using file system. Some research prototypes use a commercial database to support hypertext. The Intermedia system developed in Brown University uses the commercial database, INGRES. Intermedia provides users with a way of controlling the increased complexity of the hypertext environment. The Intermedia project is a big and long project. It is not our aim to present all the technical characteristics of Intermedia in this paper. But, we are encouraged by the Intermedia approach in that they are now moving towards using an object oriented database. ENCORE. Smith in his paper[21] argues why they used an object oriented database system to support Intermedia. We shared the same ideas. That is why we chose DAMOKLES when we started this project even though we have a commercial relational DBMS at that time.

Lange[22] developed a hypertext system to support the Program Development Environment (PDE). A complete description of the data model of this approach which is described in VDM can be found in [23]. The main contribution of Lange's work is that the traditional hypertext data model such as node, anchor, link, hyperspace and so on can be formally modelled and fully supported by using the mechanisms of an OODBMS.(3) The InterSect approach is different from Lange's in two aspects: first, the data model is different. The main difference is the definition of a structured node. In Lange's paper[22], he described that "... It should be emphasized that it is not the nodes and networks themselves that are organized in a structure...". Whereas in InterSect approach, it is a truely structured node which can contain subnodes and relationship between subnodes. Second, there is no version support in Lange's work. This is because ONTOS does not provide any version management mechanism. In InterSect, since DAMOKLES has a version management mechanism, InterSect can support both versioned structured nodes and versioned links.

Ishys, an Intelligent Software Hypertext System, is another system using a relational DBMS to support hypertext[2]. In Ishys, the database is only used to manage the relationships between Unix files which act as the hypertext nodes to store information. The Unix directory is chosen to represent the composition of nodes. The significant feature of Ishys is that Ishys first formally described the hypertext data model by using a mathematical method. We benefit from Ishys approach in particular by using set theory to describe the hypertext data model. This is one of the reasons that we choose $\mathrm{Z}$ to define InterSect data model although $\mathrm{Z}$ has its own many significant characteristics[7]. Another difference between Ishys and InterSect is that InterSect supports versioned links whereas Ishys does not.

\section{Conclusion}

The first prototype of InterSect has been developed using software documentation as an example. More work is needed for the future. This includes adding graphic functions in the Frame editor. We also would like to test InterSect in some other application areas such as system analysis or office automation. Our experience of using an object oriented database is that it was not as easy to use as a full relational database. This was mainly due to the lack of a comprehansive data dictionary to describe the schema and the lack of a high level query language. However, we found that the additional function provided of a version mechanism. support for complex objects and navigation links to other objects more than made up for the difficulties encountered. This is surpported by the experience of Lange's research[22].

(3)The OODBMS be uses is ONTOS OODBMS. 


\section{Acknowledgements}

This research was initially supported by British Admiralty Research Establishment. Special thanks to Dr. Ian Toyn for his active suggestions to improve the performance of Insersect and his $\mathrm{Z}$ type checking tool - CADIZ for type checking the $\mathrm{Z}$ in this paper.

\section{References}

1. Horowitz, Ellis. and Williamson, Ronald, "SODOS: A Software Documentation Support Environment: Its Use", Proceedings of 8th Intemational Conference on Software Engineering, pp. 8-14 (August 28-30, 1985).

2. Garg, Pankaj K. . "Information Management in Software Engineering: A Hypertext Based Approach", D.Phil thesis, Computer Science Deparment, University of Southern California (1989).

3. Bing, Wang. and Peter, Hitchcock. "InterSect. A General Purpose Hypertext System Based on an Object Oriented Database", in Proceedings of 2nd International Conference on Database and Expert Systems Applications, Berlin. Germany (August 20-24, 1991).

4. Halasz, F. G., "Reflections on Notecands: Seven Issues for the Next Generation of Hypermedia Systems". Communications of the ACM 31(7) (July 1988).

5. Apple Computer, Inc., Macintosh HyperCard User's Guide, 1989.

6. Plaisant, Catherine. "An Overview of Hyperties, Its User Interface and Data Model", Hypermedia/Hypertext and Object Oriented Darabase, pp. $52-59$ (Dec 1989).

7. Diller, Antoni., An Introduction to Formal Methods, John Wiley S Sons (1990).

8. Spivey, J. M. , The $Z$ notation: A Reference Manual, Prentice Hall (1989).

9. Date, C. J., An Introduction to Database Systems 4th Edition, Addison Wesley (1986).

10. Hall, P A V. and Papadopoulos, S., "Hypertext Systems and Applications", Information and Software Techrology 32(7), pp. $477-489$ (Maxch 1990).

11. Chen, P. P.. "The Entity-Relationship Model: Toward a Unified View of Data". ACM Transactions on Database Systems 1(1), pp. 9-36 (Mar. 1976).

12. Kamran, Parsaye., Mark, Chignell, Setray, Khoshafian and Harry, Wong., "Hypermedia", pp. 224249 in Intelligent Database: Object-oriented, Deductive and Hypermedia Technologies, John Wilky \& Sons, Inc. (1989).

13. Jones, C., Programming Productivity, McGraw-Hill, New York (1986).

14. Brown, P. J., "Hypertext: Dream and Reality", Hypermedia/Hypertext and Object Oriented Database, pp. 60-71 (Dec 1989).

15. Rada, Roy. and Diaper, Dan. "Converting Text to Hypertext and Vice Versa", Hypermedia/Hypertext and Object Oriented Database, pp. 20-35 (Dec 1989).

16. ISO8613., Information Process - Text and Office System - Office Document Architecture and Interchange Format, 1988.

17. [EE., Guidelines for the documentation of software in industrial computer systems, 1985. 
18. Abramowicz, K., Diturich, K., Gotthard, W., Hartig, M., Langle, R., Ranft, M., Raupp. T. and Rehrn. S., Damokles 2.0 - Reference Manual, FZI. University of Karisruhe (1988).

19. Dittrich. K. R., Gothard, Willi. and Lockeman, P. C., "DAMOKLES - A Database System for Software Engineering Environments", Lecture Notes on Advanced Pro. gramming Environments, pp. 353-370 (June 1987).

20. Dittrich, K. R. "The DAMOKLES Database System for Design Application: its Past, Its Present, and Its Future", pp. 150-168 in Software Engineering Environments: Research and Practice, ed. Keith H.Bennett. (1989).

21. Smith. K. E. and 2donik, S. B., "Intermedia: A Case Study of the Differences Between Relational and Object-Oriented Database Systems", OOPSLA'87 Proceedings, pp. 542-465 (Oct. 48, 1987).

22. Lange, Danny B., "Constructing a Hypertext Based Program Development Environment Using a Commercial OODBMS". Submized Paper for OOPSLA'9l (Sept. 2 1991).

23. Lange, Danny B., "A Formal Approech to Hypertex using Post-Prototype Formal Specification", Lecure notes in computer science, 428, pp.99-121 (April 17-21 1990). 


\section{APPENDIX}

In this appendix, we use a typical example taken from[18] to demonstrate the main features of the InterSect prototype. The example has the following stricture:

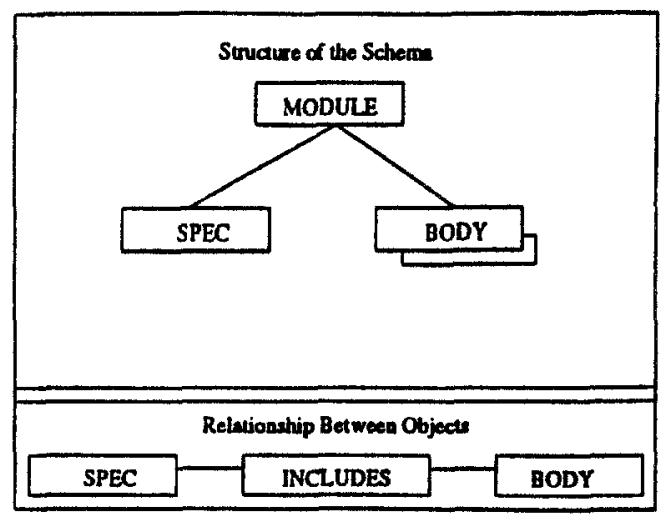

Fig 1. Structure of module

The above diagram represents the following information:

- A MODULE is a complex object type.

- A MODULE has two subtypes -- a specification, SPEC, and a source, BODY.

- The MODULE itself is a structured control node type, i.e. there is no corresponding contents definition in its type definition.

- The BODY, representing the source, is a version type.

- The SPEC represents the specification corresponding to a body.

- The SPEC and BODY are both structured node types with contents definitions attached to their type definitions. Versions of the $B O D Y$ are also contents nodes.

- A relationship type called INCLUDE is defined between the two subtypes: SPEC and $B O D Y$.

In the following sections, we will demonstrate how this complex object is maintained by InterSect.

\section{InterSect Interface}

Figure 2 shows the interface of InterSect on a Sun 3/50. The top left comer of the screen shows the continue icon, which represents the operation to continue the process. The top right comer represents the quit operation, which quits the system. InterSect also allows the user to type the Space key to continue the process or the $q$ key to leave the system. 


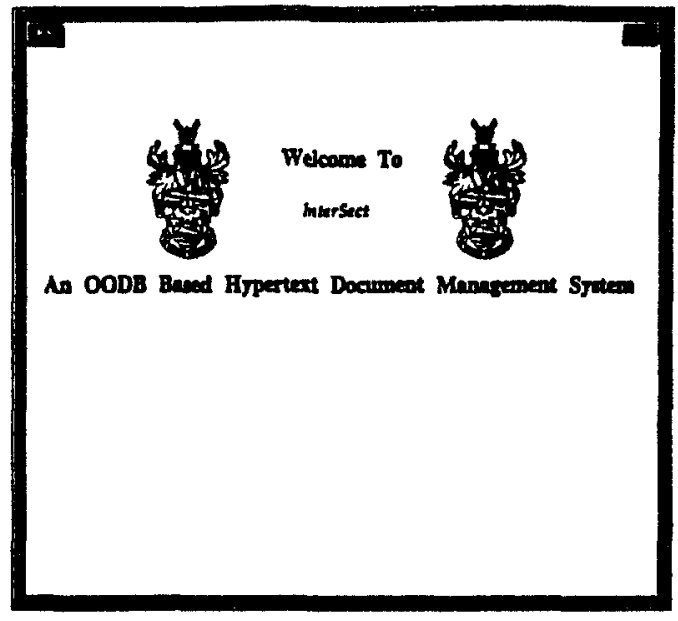

Fig. 2. InterSect interface

\section{InterSect Initial State}

Figure 3 is the first screen display of InterSect after we choose the continue operation in the figure 2.

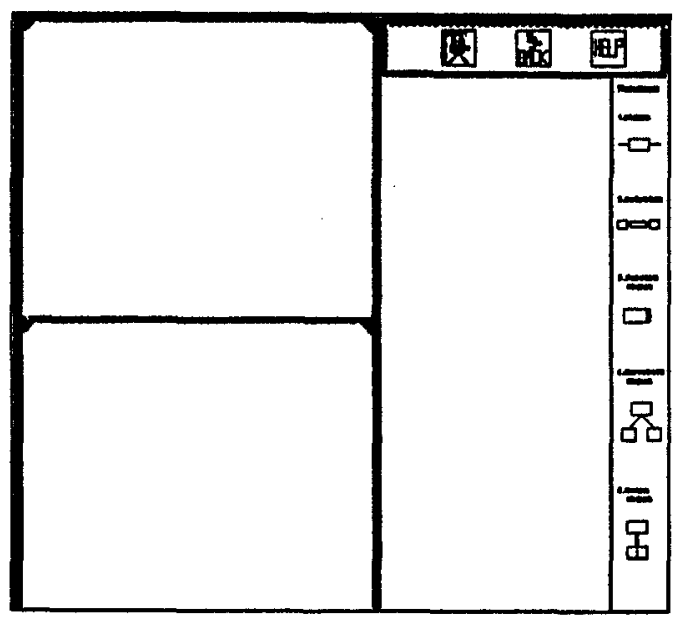

Fig. 3. Initial State of InterSect*

\section{Inputing Application Schema Definitions}

The user can edit application schema by choosing Edit Schema? which calls the Frame editor to input schema definitions or by choosing Input Schema? to directly input schema definitions.

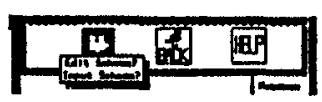

- HELP abows the information of how to use InterSect. The icows on the right side of the screes are used to display the application structure. Their meanias can be displayed if the cursor is is the corresponding icon. 
Figure 4 shows that the schema name of module, the database storing the contents of module and the owner's name are inputted into InterSect.

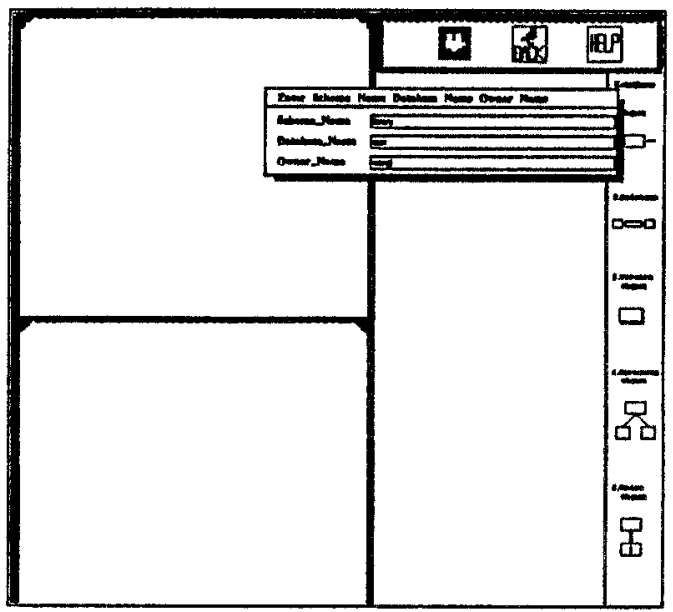

Fig. 4. Input module schema into InterSect

This information will be analysed by the InterSect schema manager. If it is correct, InterSect will continue to syntactically analyse the incoming schema definitions. If there is no syntactic errors, a graphic structure of the application will be displayed in figure 5 . Otherwise, the InterSect error reporter will report the corresponding error message.**

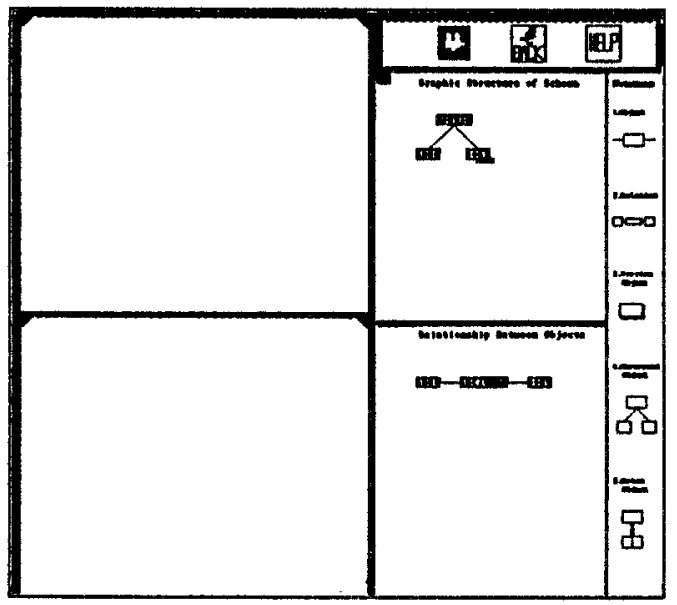

Fig. 5. Structure of the application

** If the user is not the owner of the database, for example, InterSect will repor such meastage:

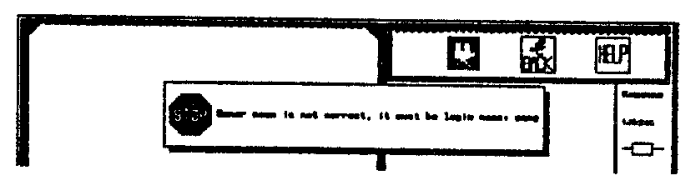




\section{Manipulations on InterSect}

InterSect allows users to browse, insert and change any object based on the defined schema definitions. In the following sections, we will illustrate the main functions of these three operations.

\subsection{Browsing a Node and Link Targets}

Each defined type can be browsed in four different ways. They are What is this, What is type, Source and Execution. What is this tells users what kind of the browsing type is. What is type tells users the type definition of the browsing type. Source shows the type definitions defined in DAMOKLES DDL. Execution lets the user further insert, browse and change the individual instances of the type. Figure 6 shows the original definition of type module defined in DAMOKLES DDL.

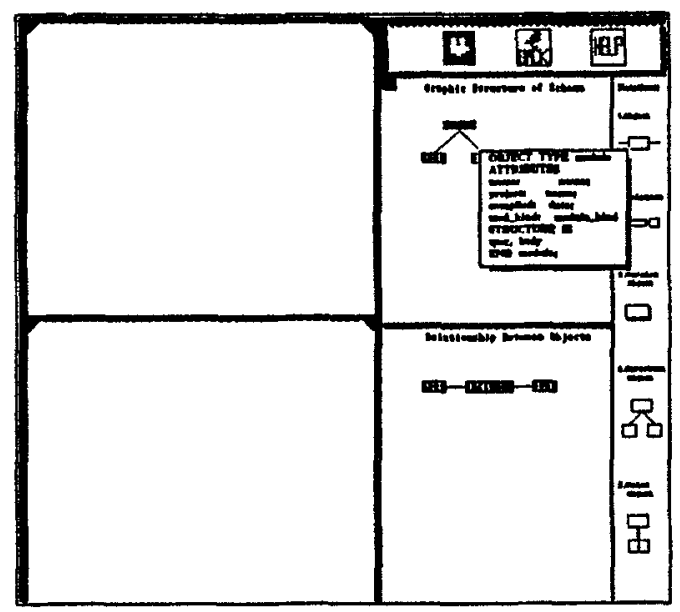

Fig. 6. Source of the type module

Browsing, inserting and changing any instance can only be possible after we choose type operation Execution. As we have described before, because nodes and links are versioning types, when browsing , inserting and changing a node, InterSect will ask the user to tell the system whether it is a generic or a specified version node that the user wants to browse, insert or change and similarly for links. Figure 7 shows a menu operation defined in the browse rectangle. The same menu will be displayed in the insert and change rectangles if inserting or changing a node. 


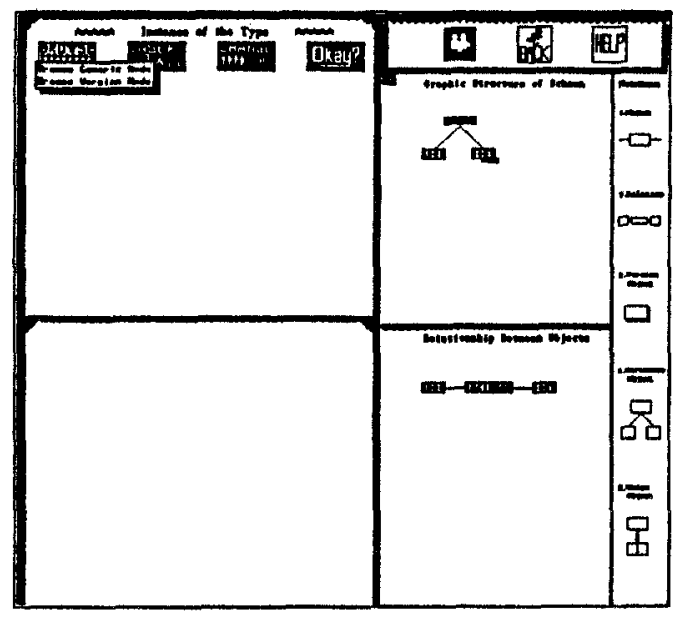

Fig. 7. Menu-operations in the browse icon

In figure 8, a found node is displayed. The text surrounded by a rectangle is the part in which the hypertext links are defined. If the linking part is selected, it will be highlighted and a type menu (Annotation Link or Referential Link) is displayed.

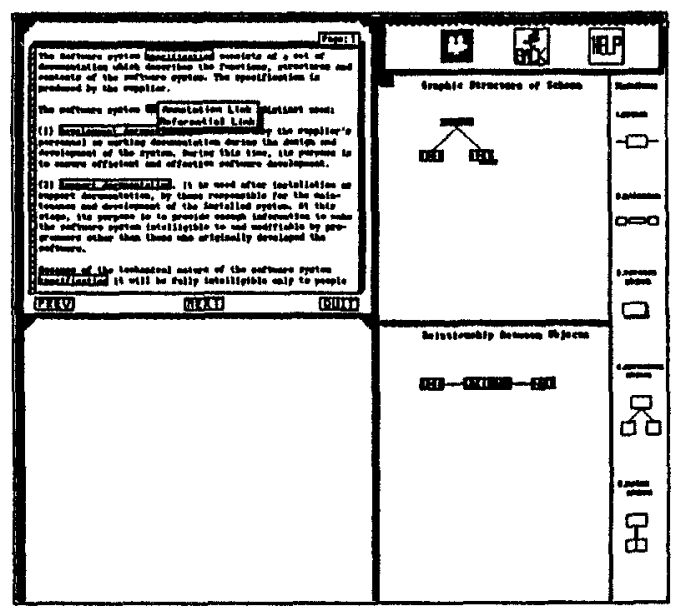

Fig. 8. A found node of type module

After choosing the link type, a version menu (Original? or Versions?) is given. The user determines whether to see the generic or a specified version link target of an annotation or a referential link, as shown in figure 9. 


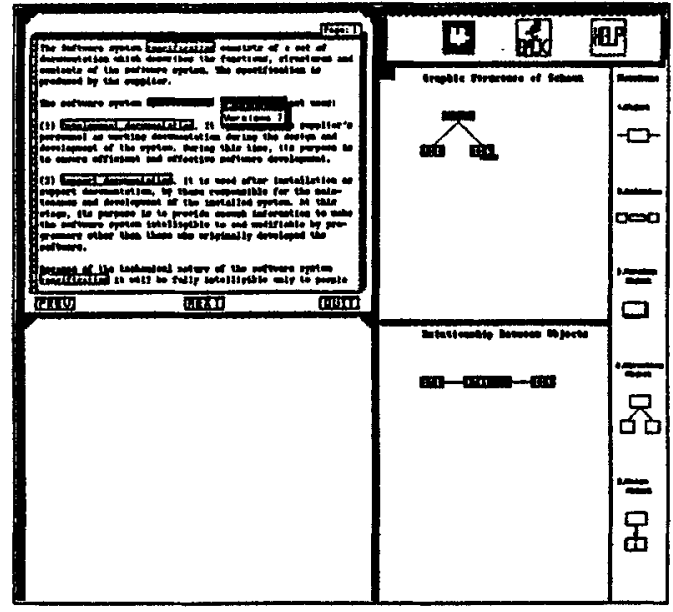

Fig. 9. The corresponding link version menu

If the highlighted part of a node has referential links defined in it, whether they are versions or a generic node, the target of the chosen link is displayed at the bottom left of the screen, as shown in figure 10 .

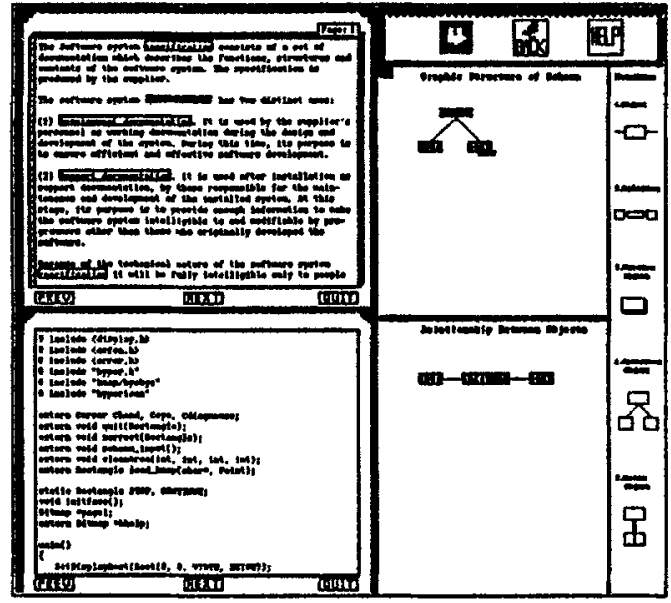

Fig. 10. A defined referential target from the chosen part

In spite of version or non-version annotation link targets, the same menu style annotation target is displayed on the screen if the annotation target exists. This is illustrated by figure 11. 


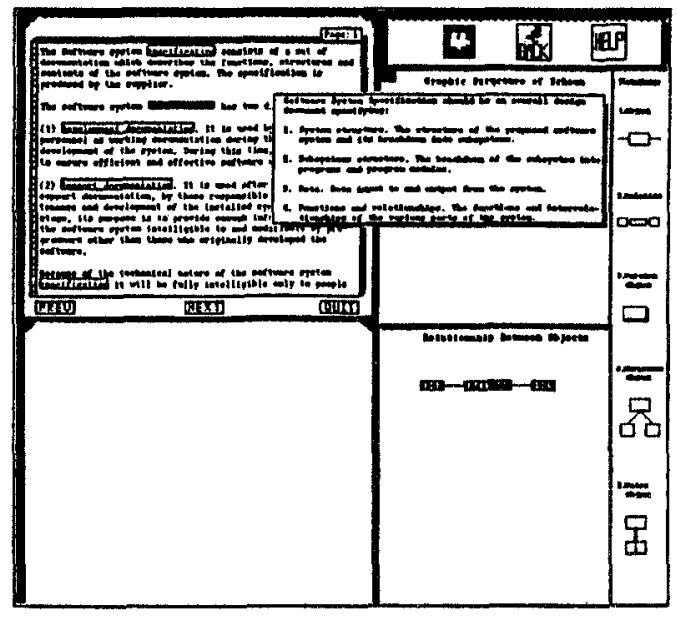

Fig. 11. A defined annotation target from the chosen part

\subsection{Inserting the Contents of a Node and Making Links}

InterSect allows the user to insert a new node or a node whose contents is based on the existing one. This is useful when we insert a version whose contents are slightly different from the original one. Figure 12 shows the situation of finding the existing node whose contents will be used as the basis for the new node.

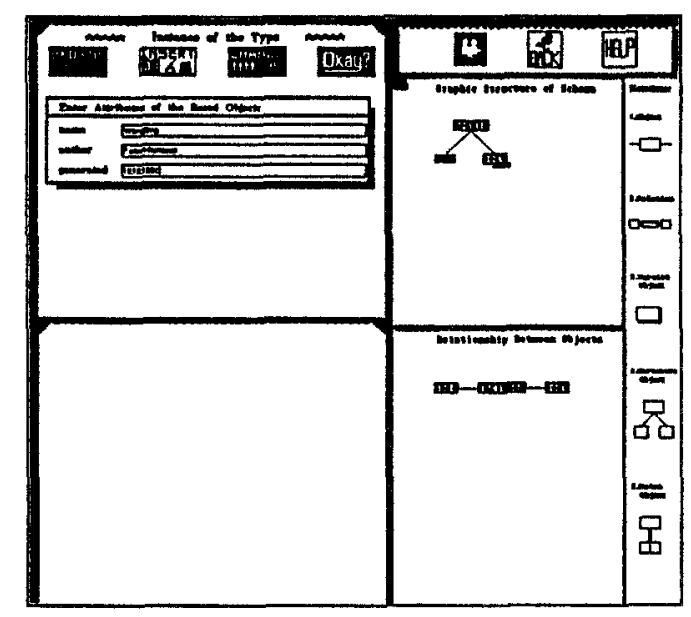

Fig. 12. Entering the attributes of a based object

If the based object already exists, the editor Frame is called and its contents are inputted as shown in figure 13. 


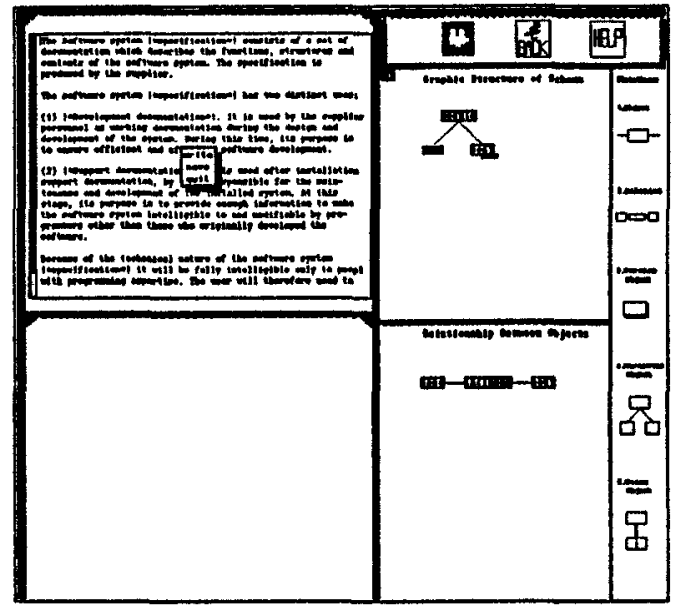

Fig. 13. Inserting the contents of a new object***

The hightlighted part in figure 13 is the source of hypertext link, i.e. the place where we will make links. If we make an annotation link, then the same editor Frame is called again in the bottom left to receive the annotation which is shown in figure 14.

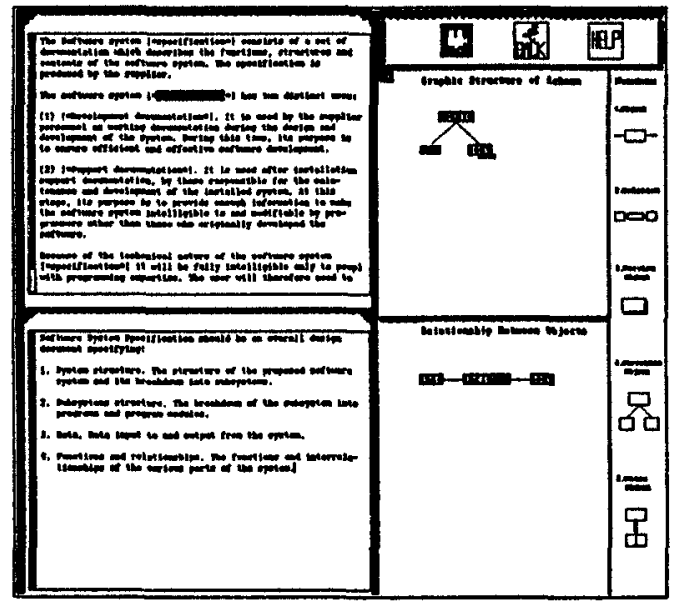

Fig. 14. Entering an annotation link

If the user wants to make a referential link, the source of the link in the top left quarter of the screen is highlighted, the same version menu as shown in figure 9 is prompted to select a versioned or non-versioned referential link.**** If this is correct then input moves to the bottom left of the screen where the attribute types of the node type which will be the referential link target are displayed as shown in figure 15.

\footnotetext{
*** The write operation is used to store the final result but not to inherit the link structure defined on the besed object. The save operation not only stores the contents but also inherits the link structure of the based object, i.c. the new object has the same link structure as its original based object but the contents are different.
} 


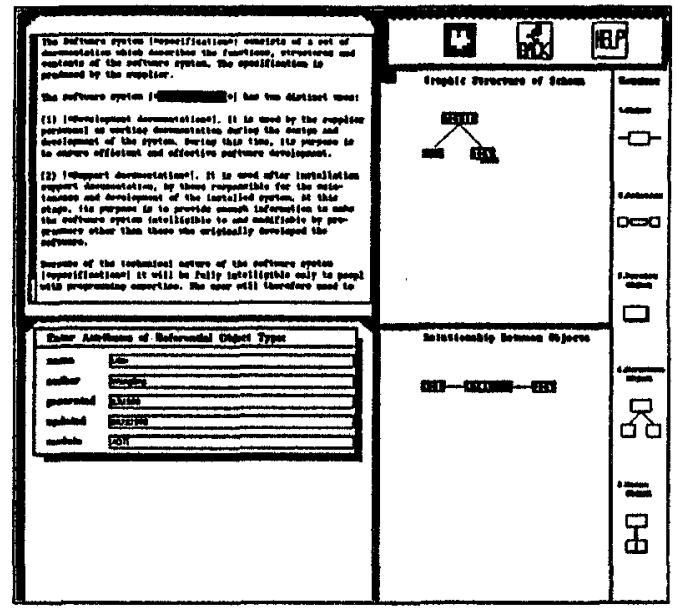

Fig. 15. Editing a referential link

\subsection{Changing the Contents of a Node}

The change operation allows the user to change the contents of a node, or to alter the relations between nodes. There are four operations defined in the CHANGE icon. These are Change General, Change Version, Delete Instance and Change Relation, as illustrated in the following picture:

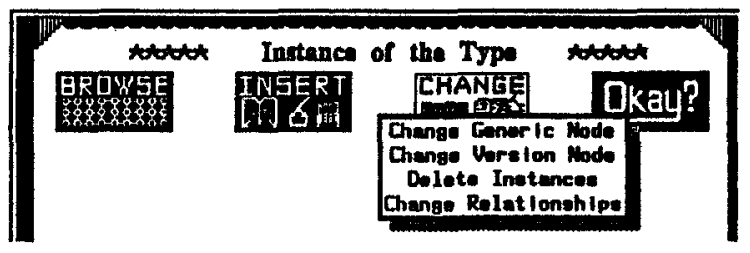

\section{Changing a Generic Node}

The procedures for changing a generic node are much like those for inserting a node. That is, firstly, an attribute menu for the node type being changed is displayed by InterSect, as shown in figure 12; Secondly, the Frame editor is called to edit the contents of the node being changed. The situation is much like that of entering the contents of a based node, which is shown in figure 13. Thirdly, the changed node is stored as a new object in the information base, although the changed object still inherits the relations defined by the old object. In addition, the old node is automatically deleted. Finally, when the user finishes changing a node or versions of a node, all the related nodes such as supernodes, sub-nodes and nodes with which it has semantic relationships are reported to the user.

\section{Changing a Version Node}

When changing a version of a node, the user is required to tell the system the generic node of the version node. This is because InterSect wants to know if the changed version node exists or not. InterSect displays the following warning message:

1*+* The situation is the same when making a version of an annotation link. 


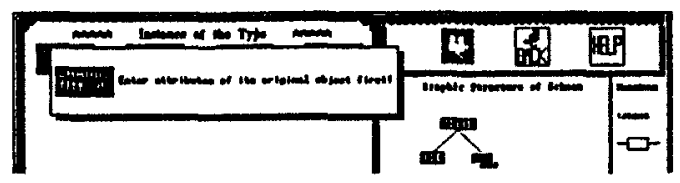

The attribute menu of the generic node is then displayed by InterSect on the screen. If this generic node exists, InterSect continues by asking the user to input the version number of the changed node.

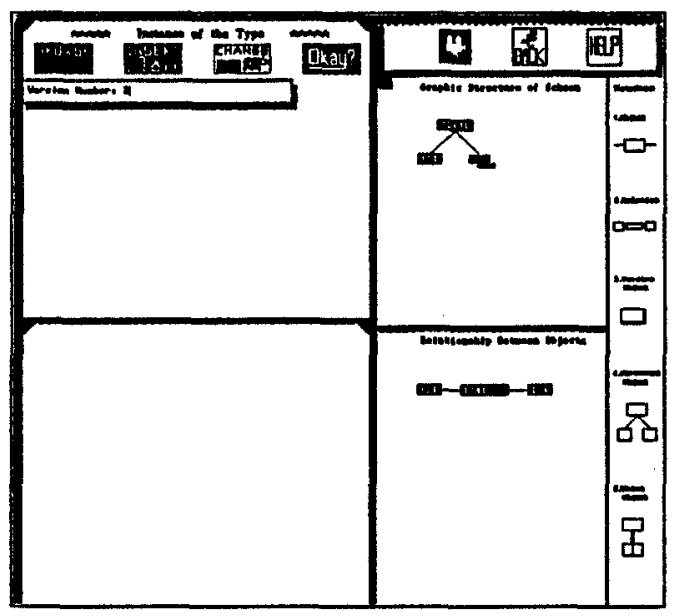

Fig. 16. Version number input for the node being changed

If this version also exists, the Frame editor is called again. The user can then edit the contents of the version node according to her/his needs. The remaining procedures for changing the contents of a version node are the same as those for changing a generic node.

\section{Changing a Structured Control Node}

Structured control nodes do not have contents. Therefore, when a structured control node is being changed the Frame editor will not be called. This implies that there is no need for us to change a structured control node. However, the user can change the attribute values of the structured control node.

\subsection{Deleting a Node}

The deletion operation is dangerous. When a node is deleted, all nodes which have either a structural or semantic relationship with it will be deleted automatically by InterSect in order to keep the semantic consistency. A sub-menu of Delete Instance is prompted when deleting a node:

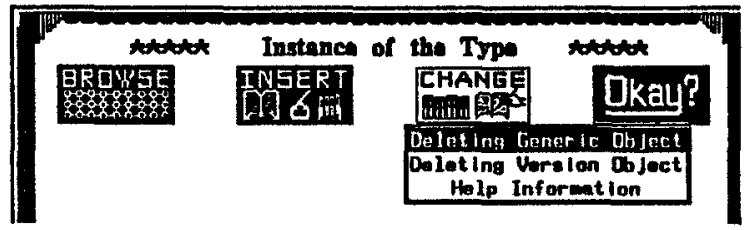


If a generic node is deleted, the attribute menu of the generic node is displayed on the screen. If deleting a version node, its generic node type menu is displayed first to receive the generic node information. If the generic node exists, the version number is required by InterSect. The Help Information shows the result of the deleting operation.

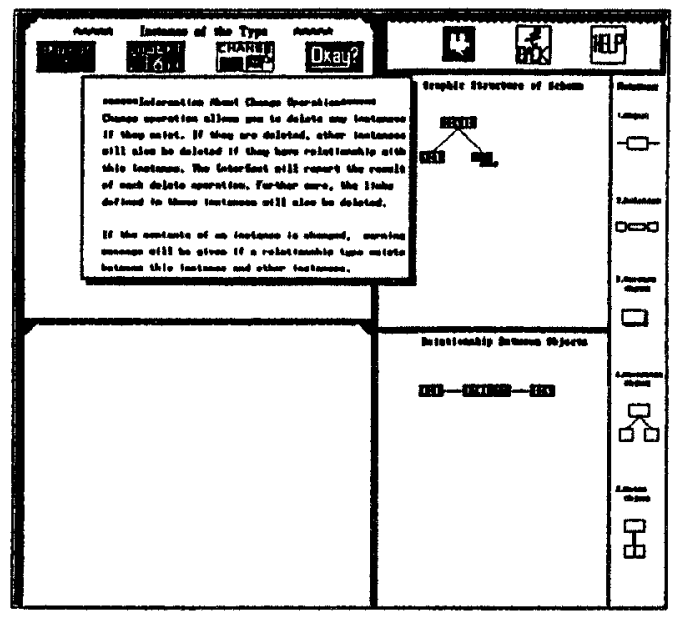

Fig. 17. Warning information about deleting a node

Therefore, we should be careful if we decide to delete a node, particularly if this node is related to other nodes.

\subsection{Changing Relations}

When the Change Relation operation is chosen, a sub-menu is displayed on the screen:

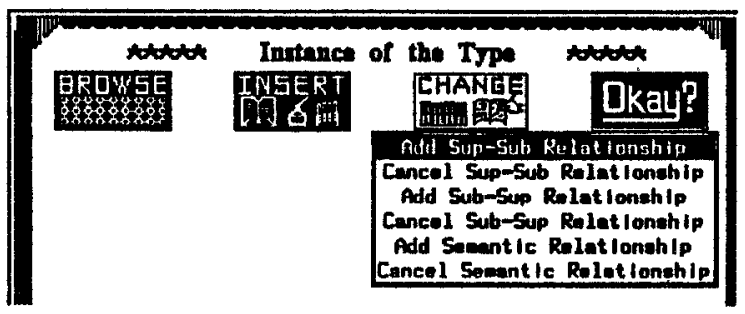

Change Relation is the operation which alters relationships of a node. The relationships of a node are either structural, such as a super-sub relation or a sub-super relation, or semantic, such as the INCLUDE between nodes SPEC and nodes BODY. There are therefore two different situations covered by the change operation:

\section{(1) Add or Delete a Structural Relation between two Nodes}

A structural relation can be added or deleted between two nodes if the types of these two nodes have a structural relationship, such as MODULE related to SPEC or MODULE related to BODY. The operations: Add Sup-Sub Relationship, Cancel Sup-Sub Relationship, Add Sub-Sup Relationship and Cancel Sub-Sup Relationship are defined to achieve this aim. 


\section{(2) Add or Delete a Semantic Relation between two Nodes}

A semantic relation such as INCLUDE between two nodes can be added or deleted by choosing the operations Add Semantic Relationship or Cancel Semantic Relationship, as long as these two nodes belong to the types which have such a relationship. For example, if we want to add a sup-sub relation between a node which belongs to the type BODY and a node which belongs to the type MODULE, we choose the Add Sup-Sub Relationship operation. A menu of the supernode is displayed by InterSect, and the attributes of the super object are input as shown in figure 18.

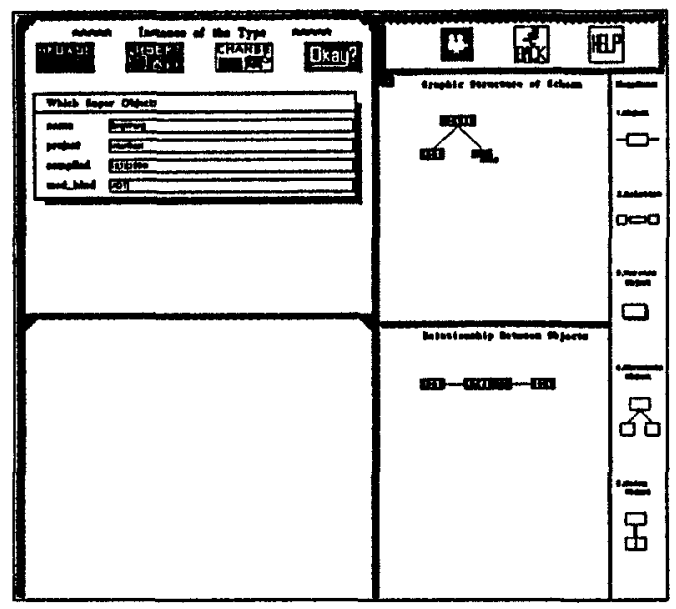

Fig. 18. Importing a supernode

If this super node exists then a menu of the sub-object is displayed, and the attribute values of the sub-node are also input into InterSect. Furthermore, the result of changing is reported by InterSect as follows:

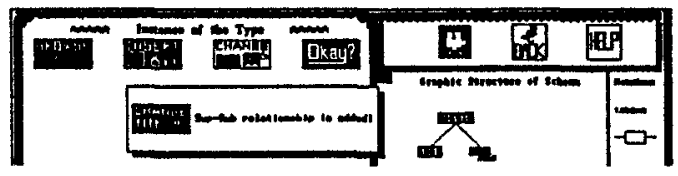

Otherwise, InterSect reports the following message if the nodes already have such a relation.

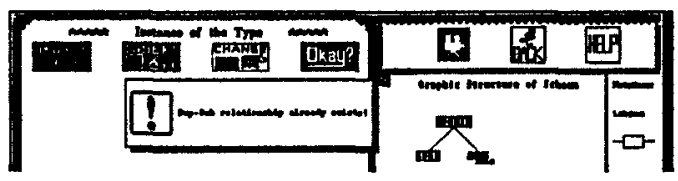

The rest of the operations are the same as those we have described above, except that different attribute menus are displayed by InterSect according to the different situations. 


\subsection{Operations on Relations}

When a relationship type is defined between two node types, InterSect uses this information to remind or wam the user when s/he inserts, browses or changes any node that has such a relation. InterSect allows the user to browse the definitions of a relationship type by displaying a menu operation on each relationship type, as shown in figure 19.

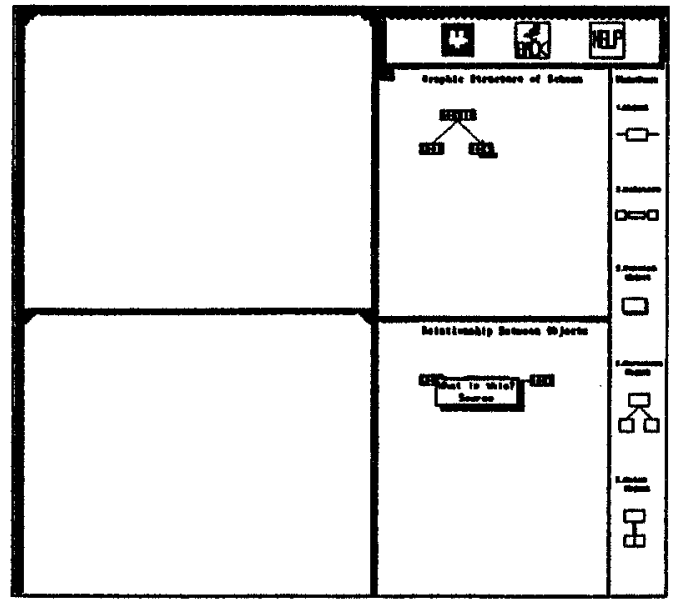

Fig. 19. Operations on the relationship type

What is this tells the user the meaning of the chosen relationship type. Source shows the DAMOKLES DDL definitions of the relationship type.

The structured node types related by the relationship type cannot be browsed. However, InterSect informs the user what $s /$ he can do next if s/he moves the cursor in the structured node type icons. The following example shows what happens if the user wants to browse $B O D Y$.

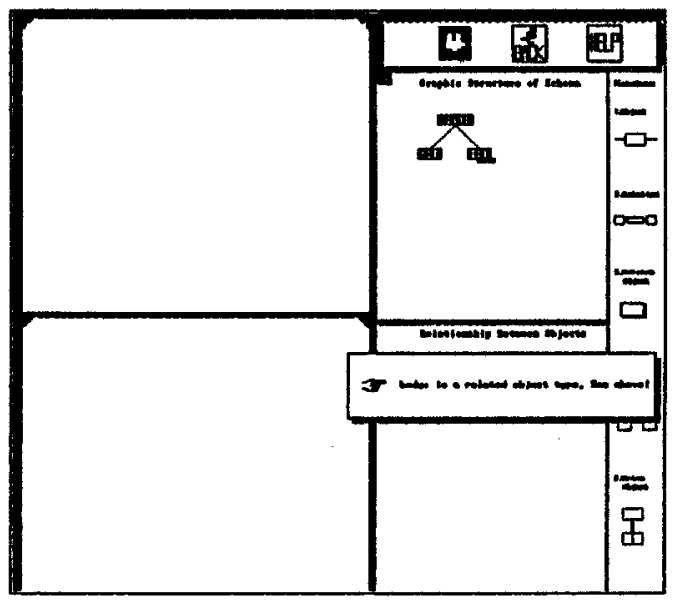

Fig. 20. The help message of the related type 